\title{
EDITORIAL
}

\section{Current Perspectives on Neurological Diseases: Experimental Models, Mechanisms of Disease and Treatments}

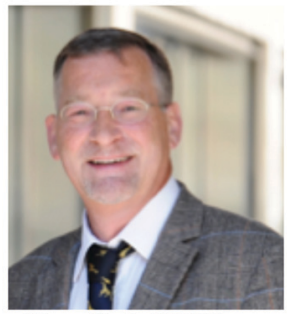

Hans Rudolf Widmer
The diseased or damaged brain has only a limited regenerative capacity which is mainly of functional nature. Effective therapies are still missing for a vast number of pathological conditions of the Central Nervous System (CNS). These usually devastating diseases have a major impact on quality of life and are associated with high socioeconomic costs. Due to increasing life expectancy and a higher prevalence of neurodegenerative and neurovascular pathologies in the elderly population, these disorders will become even more important for our society in the future and there is a need for the development of new, adequate treatment options [1].

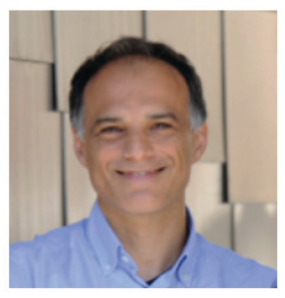

Stefano Di Santo

This thematic issue provides detailed insights into important neuropathological processes, advances in the development of strategies for disease intervention and highlights the current lack of understanding that still need to be addressed.

Parkinson's Disease (PD) is a neurodegenerative disorder mainly characterized by the loss of dopaminergic neurons in the substantia nigra pars compacta, eventually leading to a depletion of dopamine in the striatum. The progressive loss of dopamine leads to the cardinal motor symptoms in PD which are resting tremor, bradykinesia, hypokinesia, and muscle rigidity. The selective vulnerability of dopaminergic neurons against various insults, including oxidative stress, is a significant characteristic of age-related degenerative disorders. Up to now, it is not fully understood how aging interferes with the physiology of these affected neurons and how this results in age-related functional deficits. The discovery of the dopamine precursor L-dopa (3,4dihydroxyphenylalanine) opened a new area for the treatment of the motor symptoms. While L-dopa therapy provides adequate alleviation of the symptoms for several years, the long-term treatment is complicated by progressive disability and development of severe side effects such as dyskinesias, ON/OFF-periods, and hallucinations.

In their review, Bogetofte et al. [2] summarize the history and status of L-dopa treatment for PD and carefully discussed advantages and disadvantages as compared to other available therapeutics.

PD is generally categorized as a movement disorder. More recently however, it has been recognized that PD patients suffer a range of non-motor symptoms. These include neuropsychiatric symptoms which are likely associated with the dysfunctional nondopaminergic pathways occurring in PD. In this respect, Impulse Control Disorders (ICD) like hypersexuality and compulsive use of dopaminergic medication have a negative impact on the quality of life of both the patients and their relatives. Notably, there is a lack of defined strategies for the management of ICD and the only acclaimed strategies are a reduction or discontinuation of dopamine agonists and levodopa, leading thereby often to a deterioration of motor symptoms. Subthalamic deep brain (STN-DBS) stimulation has been used over the past years as a safe and effective treatment, mainly in younger patients with disabling motor complications due to levodopa treatment. The situation in patients with ICD, however, is not yet perspicuous.

In their review, Amstutz et al. [3] describe the outcome of STN-DBS based on retrospective, prospective and randomizedcontrolled studies. They conclude that ICDs improve after STN-DBS in most patients and that persisting new-onset ICDs induced by STN-DBS are rare, however, the underlying mechanisms need to be further investigated. Importantly, they propose that other non-motor symptoms, e.g. sleep disorders, depression and apathy, should also be used as secondary outcome parameters in studies addressing the impact of STN-DBS as a management strategy for ICDs.

In vitro studies on dopaminergic neurons are indispensable for drug screening and the investigation of the mechanisms of neurodegeneration in PD. Accordingly, the neuroblastoma cell line SH-SY5Y is frequently used for this purpose because of the ease of handling the human origin. Importantly to note, a detailed description of the differentiation protocols is missing in most of the studies and there is a lack of consensus about the phenotypic traits obtained. A comprehensive and quantitative evaluation of the modulation of phenotypical markers, however, is essential to allow for a proper interpretation of the results. Moreover, in order to obtain a high number of cells with a dopaminergic phenotype an optimized differentiation protocol is needed.

In their experimental article, Ducray et al. [4] describe a thorough phenotypic and morphological characterization of SHSY5Y in relation to different differentiation protocols. The authors report an enormous variation of marker expression depending on the culture conditions tested. These results are of great importance for pharmacological and disease modelling studies.

Scheller Nissen et al. [5] in depth describe the current knowledge, disease mechanisms, diagnosis and treatment of Autoimmune Encephalitides (AE). AE comprise an astounding number of diseases characterized by antibodies against neuronal synaptic and cell surface antigens. Many of the disease-causing antibodies present as limbic encephalitis which goes along with memory impairment, psychiatric features and epileptic seizures. In their review, the authors focused mainly on the two major subtypes, i.e. NMethyl-D-Aspartate receptor encephalitis and voltage-gated potassium channel complex encephalitis as the treatment options are basically the same regardless of the antibody type. While much information on clinical features, patho- 
physiology and treatment has been gathered over the last years, present treatment regimens are still based on knowledge from other antibody-mediated neurological disorders. Hence, they propose that multicenter randomized clinical trials need to be conducted to find new optimal treatment procedures. This might also be relevant for the discovery of biomarkers to monitor the efficacy of the therapy applied.

The formation of fibrotic scars is a physiological response to tissue injuries in the CNS and the Peripheral Nervous System (PNS). Current evidence indicates that fibrosis is involved in the inhibition as well as in support of repair mechanisms in the nervous tissue. In their review, Ghosh et al. [6] illustrate the cellular and molecular mechanisms underlying the development of fibrosis which follows spinal cord and peripheral nerve injuries. A better understanding of the cascade of events associated with fibrosis would enable to steer the tissue response to injuries towards restorative processes. This knowledge is thus essential to improve the efficacy of therapeutic interventions for nerve regeneration in CNS and PNS.

Glioblastoma Multiforme (GBM) is considered the most aggressive and common primary central nervous system tumor in adults. Unfortunately, despite the advances in surgical procedures, radiotherapy, and chemotherapy GBM remains an incurable disease with remarkably poor prognosis. A variety of innovative treatments have been introduced to meet the demand to improve current therapies and increase patient survival. In this context, Tumor Treating Fields (TTF) is a promising therapeutic tool for the treatment of GBM. Despite TTF has entered the clinical practice, its mechanisms of action are not entirely clear. Increasing evidence suggests that TTF exert a variety of effects in addition to the inhibition of mitotic spindles formation and cell membrane rupture in the highly proliferating GBM cells.

In their review, Kissling and Di Santo [7] have screened the literature addressing the mechanisms of action of TTF and emphasized the effects on cell physiology which have so far remained in the background but are fundamental to overcome the resistance of GBM to therapies. The resulting message is that future studies on TTF should also focus on the effects on immunity, on cell migration and angiogenesis inhibition. Harnessing these effects especially in combination with current treatments as chemotherapy or radiotherapy might lead to the development of new therapeutic approaches for GBM.

Adequately characterized model systems and clinical studies are needed to improve the knowledge on the mechanisms and underlying causes of neuropathological disorders. Given that most interventions only start when the neuropathological processes are already advanced, a detailed analysis of potential biomarkers needs to be assessed, allowing for earlier interventions. Future research involving international multicenter randomized clinical trials are warranted to elucidate optimal treatment regimens.

\section{REFERENCES}

[1] Andres RH, Meyer M, Ducray AD, Widmer HR. Restorative neuroscience: concepts and perspectives. Swiss Med Week 2008; 138(11-12): 155-72.

[2] Bogetofte H, Alamyar A, Blaabjerg M, Meyer M. Levodopa therapy for Parkinson's disease: history, current status and perspectives. CNS Neurol Disord Drug Targets 2020; 19: doi: 10.2174/1871527319666200722153156.

[3] Amstutz D, Paschen S, Lachenmayer ML, Maradan-Gachet ME, Deuschl G, Krack P, Debove I. Management of impulse control disorders with subthalamic nucleus deep brain stimulation in Parkinson's disease. CNS Neurol Disord Drug Targets 2020; 19: doi: $10.2174 / 1871527319666200720105553$.

[4] Ducray AD, Linda WL, Herren F, Widmer HR, Mevissen M. Quantitative characterization of phenotypical markers after differentiation of SH-SY5Y cells. CNS Neurol Disord Drug Targets 2020; 19: doi: 10.2174/1871527319666200708132716.

[5] Scheller NM, Ryding M, Meyer M, Blaabjerg M. Autoimmune encephalitis: current knowledge on subtypes, disease mechanisms and treatment. CNS Neurol Disord Drug Targets 2020; 19: doi: 10.2174/1871527319666200708133103.

[6] Ghosh N, Kalbermatten D, Madduri S, Guzman R. Fibrosis and regulation of nerve regeneration in the peripheral and central nervous systems. CNS Neurol Disord Drug Targets 2020; 19: doi: 10.2174/1871527319666200726222558.

[7] Kissling C, Di Santo S. Tumor treating fields-behind and beyond inhibiting the cancer cell cycle. CNS Neurol Disord Drug Targets 2020; 19: doi: 10.2174/1871527319666200702144749.

\section{Dr. Hans Rudolf Widmer}

Guest-Editor: CNS \& Neurological Disorders - Drug Targets

Department of Neurosurgery,

Neurocenter and Regenerative Neuroscience Cluster

Bern University Hospital, University of Bern

CH-3010 Bern, Switzerland

Tel: + $41(0) 316322770$

E-mail: hanswi@insel.ch
Dr. Stefano Di Santo

Co-Guest Editor: CNS \& Neurological Disorders - Drug Targets

Department of Neurosurgery,

Neurocenter and Regenerative Neuroscience Cluster

Bern University Hospital, University of Bern

CH-3010 Bern, Switzerland 